These results suggest that multiple criteria are used in selection of simultaneously available inputs. On the basis of these criteria, items may be fed through a single channel, as Broadbent originally proposed.

I thank I. Smith for running the subjects, and L. R. Boutler for discussing the results.

T. M. BLOOMFIELD

Department of Psychology,

York University,

Downsview, Ontario

Received July 14; revised November 19, 1971.

1 Broadbent, D. E., Perception and Communication (Pergamon, London, 1958).

2 Triesman, A., Amer. J. Psychol., 77, 206 (1964).

3 Cherry, C., J. Acoust. Soc. Amer., 25, 975 (1953).

${ }^{4}$ Deutsch, J. A., and Deutsch, D., Psychol. Rev., 70, 80 (1963).

5 Treisman, A., Brit. Med. Bull., 20, 12 (1964).

6 Paiermo, D. S., and Jenkins, J. J., Word Association Norms (University of Minnesota Press, 1964).

\section{Relationship between Stability and Connectedness of Non-linear Systems}

Gardner and Ashby ${ }^{1}$ have studied the relation between the probability of stability $p(S)$ and the extent of connectedness ("connectance") $C$ of large linear dynamical systems. They found a very interesting on-off-type (numerical) result: for reasonably large systems (number of components $n \geq 10$ ) there is a critical value, $C_{c}$ of $C$, below which the system is almost certainly stable, but above which it becomes almost certainly unstable.

In this communication we outline the effect of non-linearity on this striking $p(S)-C$ relationship; the general non-linearity of realistic models of physical systems lends particular importance to such an undertaking.

Let the vector function $x(t)=\left(x_{1}(t) \ldots, x(t)\right)$ represent the state of the linear system at time $t$. The time development of $x(t)$ is given by

that is

$$
\dot{x}(t)=A x(t)
$$

$$
\dot{x}_{i}(t)=\sum_{j=1}^{n} a_{i j} x_{j}(t), j=1, \ldots, n
$$

The system is assumed to be autonomous, that is, the $a_{i j} \mathrm{~s}$ are constants independent of $t$.

The connectance $C$ of the system (1) is defined as the fraction of off-diagonal elements $a_{t j}$ of $A$ that are non-zero. Thus $0 \leq C \leq 1$. For $C=0$ the system is completely disconnected, and (2) separates into $n$ independent, decoupled equations.

The stability (uniform asymptotic stability) $S$ of the linear system (1) can be related to the eigenvalue distribution of $A$ : if all eigenvalues $\lambda_{i}$ satisfy $\operatorname{Re}\left(\lambda_{i}\right)<0$, then (1) is stable, otherwise it is unstable ${ }^{2}$.

The $p(S)-C$ relationship was discovered in the following manner. (1) A value for $n$ was chosen. (2) The $n$ diagonal elements $a_{i t}$ of $A$ were selected randomly from a uniform distribution in $(-1.0,-0.1)$, that is, the individual $x_{i}$ were assumed to be intrinsically stable. (3) The $x_{i}$ s were then coupled by generating $n(n-1) C$ non-zero off-diagonal elements randomly from a uniform distribution in $(-1 ., 1$.). This completed the construction of $A$. (4) $A$ was then diagonalized and tested for stability. (5) For a given $C$ steps $2-4$ were repeated a large number of times; the fraction of random matrices found stable was then defined as (a measure of) the probability of stability $p(S)$. (6) Steps 1-5 were repeated for different $n$ values.

In general $p(S)$ decreases with increasing $C$. The larger $n$, the greater the rate of decrease. The surprising feature is the step-function-like drop of $p(S)$ from 1 to 0 at some critical $C$ value $C_{c}$ for $n \geq 10$. Gardner and Ashby conjecture that for all large complex dynamical systems such discontinuous behaviour is to be expected.

The influence of non-linearities on the $p(S)-C$ relation will be analysed in two stages: $(a)$ Effect on $S$; $(b)$ effect on $C$.

(a) Consider the non-linear autonomous system

$$
\dot{x}_{i}=\sum_{j=1}^{n} a_{i j} x_{j}+Y_{i}\left(x_{1}, \ldots, x_{n}\right), i=1, \ldots, n
$$

where we assume that the $Y_{t} s$ are convergent power series in $x_{i}$, beginning with terms of at least the second degree. Then one has the following theorem of Liapunov ${ }^{3}$.

(i) If (3) is stable for $Y_{i}=0$, all $i$ (that is, if (1) is stable), then the equilibrium $\left(x_{i}=0\right.$, all $\left.i\right)$ is asymptotically stable whatever the terms $Y_{t}$ are. (ii) If (1) is unstable (has at least one $\lambda$ with $\operatorname{Re}(\lambda)>0)$ the equilibrium is unstable whatever the $Y_{i}$ are. (iii) If (1) has some roots $\lambda$ with $\operatorname{Re}(\lambda)=0$ but none with $\operatorname{Re}(\lambda)>0$, then the terms in $Y_{i}$ can be chosen such that they have either stability or instability.

This theorem enables us to determine the stability of a class of non-linear systems (3) provided the stability of the precursor linear system (1) is known.

(b) In trying to understand the effect of non-linearity on $C$ one is faced with the problem of defining $C$ unambiguously. (This is not trivial. Even in the linear case one makes the tacit assumption that the $x_{t}$ s are physically well defined and meaningful components of the interacting system, that is that the coupling of these components does not destroy their physical identity; otherwise, purely mathematical transformations $x_{i}^{\prime}=T\left(x_{i}\right)$ can be constructed that would change $C$ arbitrarily.) We ignore this dilemma and assume that the definition of $C$ used for the linear system is also applicable in the non-linear case. We write

$$
Y_{t}\left(x_{1}, \ldots, x_{n}\right)=\sum_{j=1}^{n}\left[\sum_{k=1}^{n} b_{i k} x_{k}{ }^{j}\right] x_{j}, \text { all } i
$$

where the $b_{i k}$ s are constant. Then (3) becomes

$$
\dot{x}_{i}=\sum_{j=1}^{n}\left\{a_{i j}+\sum_{k=1}^{n} b_{i k} x_{k}{ }^{j}\right\} x_{j}, \quad i=1, \ldots, n
$$

Assume that the linear constituent of $(4)$ is diagonal and stable (that is, $C=0, a_{i j}=-\delta_{i j} a_{i i}, a_{i j}>0$ ). Clearly, Liapunov's theorem ensures that by different choices of the $b_{i k}$ one can vary $C$ between 0 and 1 , without affecting the stability of (4) ! Consider now a collection of linear systems (generated via the Gardner-Ashby procedure) which is characterized by a particular $(p(S), C)$ pair. According to our procedure, we can add non-linear terms to each linear system in the collection without affecting its stability either way (i) and (ii) of Liapunov's theorem; we assume that the probability of (iii) being operative is negligible). At the same time, we can change the $C$ values at will. In particular, the $\left(p(S)=1, C<C_{c}\right)$ pairs can be changed to $\left(p(S)=1, C_{c} \leq C \leq 1\right)$.

These results indicate that in the presence of non-linearity the concept of critical connectance may be meaningless in its present form. This conclusion does not deny either the existence or the importance of such a concept; it merely indicates that a more fundamental definition of connectance is needed. Perhaps an approach similar to Kauffman's ${ }^{4}$ will be more successful.

D. N. G. is an NRCC postdoctoral fellow.

\section{R. L. SOMORJAI \\ Dipendra N. Goswami}

Division of Chemistry, National Research Council of Canada, Ottawa K1A OR6

Received December 2, 1971.

1 Gardner, M. R., and Ashby, W. R., Nature, 228, 784 (1970).

2 Gantmacher, F. R., Applications of the Theory of Matrices, ch. $v$ (Interscience, New York, 1959).

${ }^{3}$ Minorsky, N., Theory of Nonlinear Control Systems, ch. 4 (McGraw-Hill, New York, 1969).

4 Kauffman, S. A., J. Theoret. Biol., 22, 437 (1969). 